\title{
Inmersión educativa de e-sport. Del contexto lúdico al mundo educativo
}

Enviado: 22 de junio de 2021 / Aceptado: 14 de septiembre de 2021 / Publicado: 31 de diciembre de 2021

LUIS MIGUEL MATEOS TORO

Universidad Internacional de La Rioja luismiguel.mateos475@comunidadunir.net

iD $\underline{0000-0002-6747-6295}$

LAURA ABELLÁN ROSELLÓ

Universidad Internacional de La Rioja

laura.abellan@unir.net

0000-0003-3009-9024

DOI 10.24310/IJNE.8.2021.12900

\section{RESUMEN}

La investigación trata de conocer en qué medida influye el profesorado que impartió clase mientras estudiaba estudios secundarios y/o estudios secundarios en estudiantes que practican e-sports. La muestra recogida son jugadores que compiten al FIFA21 formada por 30 personas, se ha utilizado un análisis no paramétrico de una muestra, una regresión lineal, una correlación, estudios descriptivos y ANOVA de un factor. Son utilizadas las siguientes variables, la muestra de respeto y trato justo respecto a todo el alumnado, respondía con interés a las intervenciones, muestra de respeto y trato justo, organización y preparación de las clases, las explicaciones relacionadas con ejemplos, la claridad y precisión de la transmisión de contenidos, el grado de concordancia entre lo explicado con los exámenes, los días de competición, las horas fuera del entrenamiento oficial, la relación con los capitanes, las horas jugando a otras modalidades y las horas de entreno podrían influir en el abandono escolar.

\section{ABSTRACT}

Educational immersion to e-sport. From the playful context to the educational world

The research tries to know to what extent the influence of teachers who taught while studying high school and/or secondary education has on students who practice e-sports. The sample collected are players who compete in FIFA21 formed by 30 people, a nonparametric analysis of a sample, a linear regression, a correlation, descriptive studies and ANOVA of a factor have been used. The following variables are used, the sample of respect and fair treatment with respect to all the students, responded with interest to the interventions, sample of respect and fair treatment, organization and preparation of the classes, the explanations related to examples, the clarity and precision of the transmission of contents, the degree of concordance between what was explained with the exams, the days of competition, the hours outside the 
Palabras Clave: Escuelas secundarias, Fracaso escolar, Motivación, Comportamiento del maestro, deportes electrónicos, Abandono escolar. official training, the relationship with the captains, the hours playing other modalities and the hours of training could influence the school dropout.

Keywords: High schools, School failure, Motivation, Teacher behavior, e-sports, Dropout.

\section{INTRODUCCIÓN:}

El objetivo de este estudio es ver cómo afectan los e-sports en la educación, concretamente a los jugadores que compiten en Virtual Football Organization en el rendimiento escolar con las variables socialización e influencia del profesorado.

Actualmente en España, existen alumnos que están estudiando la educación obligatoria secundaria que les dedica alrededor de 2 horas al día a jugar con videojuegos y jugando a altas horas de la noche afectando en el rendimiento académico en su instituto y llevándolo a un abandono escolar.

Es también importante enfatizar que, en los juegos virtuales, no existe un contacto físico entre los compañeros de equipo o rivales y que existe diferentes roles dentro de un mismo equipo, el manager o dueño de un equipo, la persona que tiene mayor poder dentro de un equipo, capitanes el segundo nivel de poder dentro de un equipo y los jugadores. A pesar de que no se conocen entre ellos físicamente, la relación entre compañeros es intensa y la mayoría del bloque de los equipos llevan jugando juntos varios años.

Los deportes electrónicos o e-sports son competiciones virtuales de videojuegos. De forma general se trata de torneos en modo multijugador en la que cada participante compite desde su casa o de un lugar determinado. Jugando con sus compañeros en sus respectivos hogares, enfrentándose contra otros oponentes, estas competiciones han aumentado tanto en popularidad como en desarrollo de los videojuegos (Videojuegos y educación, 2020).

Dentro de la amplia gama de los e-sports, esta investigación se centrará exclusivamente en el videojuego FIFA 20 en la modalidad de CLUBES PRO dentro de la competición de Virtual Football Organization (VFO). Se trata de una liga que consta de seis divisiones en la que participan cuatrocientos treinta y cuatro equipos y seis mil novecientos cuarenta y cuatro jugadores. 
La mayoría de equipos electrónicos que compiten en VFO comienzan los entrenamientos de domingo a jueves a partir de 22:00, terminando los entrenamientos y partidos oficiales a altas horas de la madrugada.(VFO - Normativa, 2020), observando las horas de entrenamiento de los respectivos jugadores que compiten en VFO, este estudio trata de conocer cómo afecta el rendimiento académico al jugar hasta altas horas de la madrugada compitiendo con sus compañeros, requiriendo en la mayoría de los casos máxima concentración como ocurre en los deportes más convencionales teniendo clase al día siguiente. Teniendo en cuenta la necesidad de madrugar al día siguiente, no durmiendo lo recomendado, ni descansando adecuadamente para un rendimiento adecuado.

El uso de los videojuegos en España se remonta a 1980 con la entrada de la consola NES de Nintendo y SEGA de Master Sytenm hasta nuestros días.

Por otro lado, en el sistema educativo español, lleva arrastrando años con datos negativos, teniendo un grave problema de abandono escolar, deserción escolar y absentismo escolar que necesita ser estudiada en profundidad según la Organización para la Cooperación y el Desarrollo Económico (OECD, 2018). En España todavía un 21,5 \% de los jóvenes de 18 a 24 años de edad ni han completado estudios postobligatorios ni están estudiando, ostentando una tasa que dobla la media de la UE-27, situada en el 12 \% (Serrano y Soler, 2015).

Por otro lado, hay que tener en cuenta que los alumnos cursando Educación Secundaria Obligatoria le dedican 1,86 horas de lunes a viernes a videojuegos y 2,47 horas le dedica a jugar a videojuegos en fin de semana (INE, 2020).

\section{REVISIÓN LITERARIA}

\subsection{La socialización en la adolescencia}

La adolescencia es la etapa que abarca la transición de la niñez a la adultez. En esta época la socialización es el proceso por el cual el alumnado adquiere conocimientos, valores, costumbres y sentimientos que influyen en la adaptación a un comportamiento social positivo (Cava y Musitu, 2000). Por lo tanto, socializar se trata de un proceso a través del cual el alumnado aprende a diferenciar los comportamientos positivos de los negativos. Aunque este proceso de socialización comienza en la niñez, continúa hasta la adolescencia, etapa donde se hacen más relevantes otros ámbitos como el familiar, el grupo de iguales, el ámbito escolar o los medios de comunicación (Bandura, 2020). 
Por esta razón, los adolescentes suelen prestar mayor atención a la familia en ciertos temas, como cuestiones morales, económicas o educativas, mientras que dan mayor credibilidad a sus iguales en temas como la amistad, las relaciones o el tiempo libre (Smetana et al., 1993).

Por otro lado, la conducta pro social ofrece al adolescente cierto bienestar, mientras que la conducta agresiva o una conducta antisocial produce el rechazo de los demás. La importancia de este aspecto reside fundamentalmente en que los adolescentes aceptados por sus iguales, presentan una adaptación más favorable tanto en el ámbito social, como en el personal y el académico(Chen, 2006). Por lo que la conducta pro social se desarrolla en el adolescente con sus iguales, lo que se convierte en un factor de protección ante posibles problemas de conducta.

\subsection{El afecto del alumnado hacia el profesor}

Dependiendo del tipo de afecto que se tenga hacia el profesor, este puede incrementar si el afecto es positivo y cordial hacia el profesor, pero, sin embargo, puede que disminuya el rendimiento académico del alumno si el afecto entre el profesor es negativo (Galanti, 2020).

Las competencias emocionales son parte importante para la formación ciudadana, además de las competencias cognitivas (Marcelo y Luis, 2019), así que las competencias emocionales se pueden resumir en la capacidad para reconocer y dominar las propias emociones, la empatía o capacidad para sentir lo que el otro siente e identificar las emociones de los demás (Ariza-Hernández, 2017), ser competente en el manejo de las emociones permite sentirse bien, estar tranquilo y transmitir esto al interactuar con los demás, así mismo permite la solución de conflictos, la toma de decisiones de forma asertiva y por ende la solución de problemas (Sánchez y Alexander, 2019).

En la adolescencia se acometen transformaciones físicas y psicológicas que provocan que cambien su personalidad y puede verse afectado su rendimiento escolar (Blanco, 2017).

Mejorar la situación educativa supone implementar, potenciar y generalizar a todos los centros educativos, las diversas medidas que han resultado útiles para atender la diversidad, como los desdoblamientos, las tutorías individualizadas, las actividades escolares, las propuestas metodológicas interdisciplinarias y globalizadoras entre otras (Llamas, 2015).

Los problemas sociales y no solo académicos que están ocupados a los responsables políticos, profesionales de la educación, padres y madres y la ciudadanía, en general es la consecución de un sistema educativo efectivo y eficaz que proporciones a los alumnos el marco idóneo a donde desarrollar sus potencialidades, por otro lado, el indicador del nivel educativo adqui- 
rido en este estado y en la práctica totalidad de los países desarrollados y en vías de desarrollo ha sido, sigue y probablemente seguirán siendo las calificaciones escolares, a su vez, estas son reflejo de las evaluaciones y/o exámenes donde el alumno ha de demostrar sus conocimientos sobre las distintas áreas o materias que el sistema considera necesarias y suficientes para su desarrollo como miembro activo de la sociedad (Balladares y Bersabeh, 2018).

\subsection{La situación emocional del profesor}

El profesorado se encuentra en una situación compleja y delicada, se observa como se le culpabiliza de los problemas que se dan tanto en el contexto escolar, en lo que atañe a las relaciones profesorado-alumnado, así como el conflicto escolar, falta de asimilación de los contenidos curriculares entre los estudiantes o como lo referente a cuestiones de tipo social, violencia, desprestigio, adicciones, malos hábitos, todo ello propicia que el profesorado se sienta, personalmente y profesionalmente abrumado y desconcentrado con fuertes contradicciones entre sus derechos y deberes (Redó, 2017).

\subsection{Rendimiento académico y los e-sport}

Partiendo de la perspectiva de la realidad virtual, la relación entre el uso de videojuegos y los índices escolares tiene como consecuencia en presuntos efectos negativos sobre el rendimiento escolar del jugador, debido a la ausencia y a un posible desplazamiento del tiempo de estudio y de la realización de actividades escolares en el propio domicilio (Velasco y Alejandra, 2019).

Esto puede conllevar a que los usuarios van en busca de aceptación y reconocimiento, de relaciones más estables, comparado con aquellas que establecen en su vida cotidiana, se reitera la idea de que la adolescencia es vivida como una etapa de desarrollo acentuada por considerables cambios físicos, cognitivos, emocionales y sociales.

En esta etapa evolutiva por lo tanto se demanda por parte del adolescente uso de recursos personales y sociales se le plantea retos, generando una tensión por alcanzarlos. Si el adolescente percibe esta etapa como estresante, sin percatarse de sus logros, puede frustrarse y puede optar por usar algunas estrategias para aliviar la tensión. Una de estas estrategias puede ser el uso excesivo de los videojuegos (Jiménez et al., 2016).

Los videojuegos ocasionan peores calificaciones producto de sus efectos sobre la asistencia a clase y realización de tareas, como argumentar que los alumnos con bajas calificaciones y con escaso seguimiento de las tareas educativas, tienden a jugar más a los videojuegos en comparación con aquellos que poseen mejor rendimiento escolar (Salguero, 2009). 
Para Rojas-Bermúdez, (2017) indica que los alumnos utilizan tanto en el aula como en sus hogares, en su tiempo de ocio, estableciendo una estrecha relación de convivencia con los monitores o pantallas. El autor indica que los videojuegos están aceptados y la percepción colectiva que se tiene en ellos es como una imagen de una actividad que forma parte del horizonte lúdico y cultural del adolescente.

\subsection{El abandono escolar y los e-sport}

A lo largo de la historia, los videojuegos han sido tratados como una supuesta enfermedad mental, sin embargo, la Organización Mundial de la Salud (OMS) recientemente ha hecho un exhaustivo análisis y determinó que el uso excesivo de los videojuegos puede causar consecuencias psicológicas, sociales y mentales para la persona que no tiene un uso de conciencia sobre ellos (Humphreys, 2019).

Los adolescentes y niños son más propensos a entrar en adicciones ya que si no tienen una buena orientación puede crear dependencia, desde la perspectiva del joven puede pensar que es algo en lo que sus padres están orgullosos con la esperanza que pueden convertirse en profesionales y/o millonarios y jugaran videojuegos sin control volviéndose unos adictos y si esto no se le pone un control desde el inicio, podría traer consecuencias muy perjudiciales en un futuro, les puede afectar cómo volverse dependientes de los videojuegos para poder ser felices, los jóvenes que ya son jugadores adictos suelen confundirse al momento de diferenciar la realidad de la ficción y suelen hacer actos que llevarían a cometer errores que les pueden dar como consecuencia la pérdida de la confianza ante los seres cercanos o cosas peores (Martinez et al., 2018)

Los videojuegos son la principal causa de pérdida de tiempo entre los adolescentes. Hay que aclarar que no solo depende la adicción a la accesibilidad, sino que existen múltiples factores que proporcionan la conducta adictiva, psicológicos, sociales, familiares, ocio entre otros (Vallejos y Capa, 2010).

\section{METODOLOGÍA}

\subsection{Tipo de estudio}

El tipo de estudio es cuantitativo, dado que facilita la posibilidad de reproducir los resultados de la muestra a una población en general, siendo una investigación cuasi experimental, debido a que no existe un grupo de control. Además, la muestra no es aleatorizada debido a 
que los jugadores de e-sports han sido elegidos de forma intencional. Por último, este estudio se enmarca dentro de los procesos de revisión y elaboración de instrumentos psicométricos, partiendo de una propuesta metodológica inmensa en la medición educativa.

\subsection{Participantes}

La muestra está compuesta de 30 personas que están compitiendo en un equipo de e-sport en la modalidad de clubes pro del videojuego FIFA 2020, 29 hombres siendo el $95 \%$ de la muestra y por 1 mujer con el porcentaje de $5 \%$, con edades que oscilan de 16 años a 51 años siendo alumnos matriculados en la educación secundaria, bachillerato, ciclo de grado medio, ciclo de grado superior, carrera universitaria y en la educación secundaria para adultos con una edad media de 24.73 años y con una desviación típica de 8.686.

\subsection{Instrumentos}

Ha sido realizado un cuestionario, que trata de 80 reactivos, 29 de los cuales son valorados con una escala de puntuación compuesta por las opciones de muy insatisfecho, insatisfecho, satisfecho y muy satisfecho, 37 son cuestiones de varias respuestas donde se debe elegir una de ellas, 8 son cuestiones personales sobre la muestra y las seis últimas preguntas están configuradas como preguntas abiertas. Por otro lado, los 80 ítems se han dividido por bloques, en el primer bloque trata de información personal y hábitos de los jugadores e-sport, en el segundo bloque se hace referencia al centro educativo, en el tercero al aula, el cuarto al profesorado, el quinto bloque hace referencia al situación personal y familiar, el sexto bloque hace referencia a la hábitos saludables, en el séptimo bloque el entorno personal, en el último bloque hace referencia el motivo de su retorno al sistema educativo.

Respecto a los indicadores de fiabilidad los autores han analizado el coeficiente de alfa de Cronbach añadiendo el estadístico total-elemento para obtener la consistencia interna de forma global. Los resultados fueron positivos, según los análisis giran en torno a un porcentaje aceptable de consecuencia interna de .786 según el modelo de Cronbach.

La realización del instrumento realizado por Sancho y Grau "Diseño y validación de un cuestionario sobre fracaso escolar en alumnado de educación secundaria de un centro de formación de personas adultas" con un análisis de fiabilidad con un porcentaje aceptable de consistencia interna de ,756 según el modelo de Cronbach (Sancho y Grau, 2013). 


\section{RESULTADOS}

Se ha realizado una regresión lineal y un análisis de correlación, por un lado, una comparación entre la relación con el mánager del equipo y con las variables independientes relacionadas las que afectan al profesorado (4.A respecto al profesorado que le dio clase en la ESO, muestra de respeto y trato justo respecto a todo el alumnado, 4.B respecto al profesorado le dio clase durante la ESO, respondía con interés a las intervenciones de los alumnos/as, 4.C respecto al profesorado le dio clase durante la ESO, muestra de respeto y trato justo respecto a todo el alumnado, 4.D respecto al profesorado que le dio clase durante la ESO, organización y preparación de las clases, 4.F respecto al profesorado que le dio clase durante la ESO , las explicaciones relacionadas con ejemplos y la realidad inmediata, 4.G respecto al profesorado que le dio clase durante la ESO, la claridad y precisión de la transmisión de contenidos e ideas en el aula de aprendizaje de los alumnos/as, 4.I respecto al profesorado que le dio clase durante la ESO, el grado de concordancia entre lo explicado y los exámenes realizados).

\subsection{Análisis de Correlación}

En la siguiente tabla se analizan las horas fuera de entrenamiento, las horas de otras modalidades, las horas de estudios y las horas de entrenamiento con su equipo, con el objetivo de correlacionar las horas dedicadas al uso de los videojuegos con las horas de estudio.

En la tabla 1, referida a las horas fuera del entrenamiento, las horas dedicadas a otras modalidades de e-sport, las horas dedicadas a los estudios y las horas de entrenamiento con su equipo existiendo correlaciones en cada una las variables analizadas al tener una significación mayor a 0.5 las horas dedicadas fuera del entrenamiento, las horas en otras modalidades, las horas de estudio y las horas de entrenamiento.

Tabla 1. Corr elación de la muestr a

\begin{tabular}{lllll}
\hline & 1 & 2 & 3 & 4 \\
\hline 1. Horas fuera del entr enamiento & 1 & $.25 *$ & $.172 *$ & $.211 *$ \\
\hline 2. Horas otr as modalidades & - & 1 & $.446 *$ & $.07 *$ \\
\hline 3. Horas de estudio & - & - & 1 & $.232 *$ \\
\hline 4. Hor as de entr enamient o & - & - & - & 1 \\
\hline
\end{tabular}


En la tabla 2, se analizan los factores relacionados sobre el profesorado la relación de las familias del alumnado con el profesorado y la práctica de actividad física del alumnado siendo concretamente estas variables analizadas, muestra de respeto y trato justo a todo el alumnado, respondió con interés a las preguntas del alumnado, las muestra de respeto y trato justo del profesorado, la organización de las clases del profesorado, la relación de la familia con el profesorado y la actividad física que practicaba al día.

Tabla 2. Correlación de la muestr a

\begin{tabular}{|c|c|c|c|c|c|c|c|c|c|}
\hline & 1 & 2 & 3 & 4 & 5 & 6 & 7 & 8 & 9 \\
\hline $\begin{array}{l}\text { 1. Muestra de respeto y trato } \\
\text { justo a todo el alumnado }\end{array}$ & 1 & $.504 *$ & $.182 *$ & $.384 *$ & $.302 *$ & $.517 *$ & $.109 *$ & $.057 *$ & $.374 *$ \\
\hline $\begin{array}{l}\text { 2. Respondía con int erés a las } \\
\text { preguntas del alumnado }\end{array}$ & - & 1 & $.129 *$ & $.490 *$ & $.227 *$ & $.406 *$ & $.364 *$ & $.316 *$ & $.158^{*}$ \\
\hline $\begin{array}{l}\text { 3. Muestra de respeto y tr ato } \\
\text { justo del pr ofesorado }\end{array}$ & - & - & 1 & $.237 *$ & $.066 *$ & $.37 *$ & $.265^{\star}$ & .000 & -.135 \\
\hline $\begin{array}{l}\text { 4. Organización de las clases } \\
\text { del pr ofesorado }\end{array}$ & - & - & - & 1 & .000 & $.117 *$ & $.419 *$ & $.537 *$ & $.341^{*}$ \\
\hline $\begin{array}{l}\text { 5. Relación de la f amilia con el } \\
\text { profesorado }\end{array}$ & - & - & - & - & 1 & $.572 *$ & $.507 *$ & $.366 *$ & $.251 *$ \\
\hline 6. Actividad física al día & - & - & - & - & - & - & 1 & $.560 *$ & $.251 *$ \\
\hline 7. Tipo de deport e realizado & - & - & - & - & - & - & - & 1 & $.243 *$ \\
\hline 8. Relación Capit án e-sports & 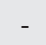 & - & - & - & - & - & - & - & 1 \\
\hline
\end{tabular}

En la tabla 2, se puede observar que existen correlaciones y significación en las variables analizadas, todas ellas relacionadas con el profesorado y la relación con los capitanes.

\subsection{Regresión lineal}

Se ha realizado un análisis de regresión para determinar el tipo grado sobre las variables independientes en qué medida están influyendo sobre las variables dependientes, permitiendo poder explicar el suceso y prediciendo acerca del futuro, siendo como variable dependiente, la relación con el capitán de su equipo de FIFA CLUBES PRO y por otro lado las nueve variables independientes relacionadas con el profesorado que le dan clase o le dieron clase cuando estuvieron en la E.S.O. 
En la tabla 3, se puede observar que el valor $\mathrm{R}$ es de .610 tiene un valor significativo, por otro lado $\mathrm{R}$ cuadrado tiene un valor .372 siendo un valor no muy alto pero sin embargo tiene un valor positivo de la variable, los resultados para realizar la regresión lineal, el valor de $\mathrm{R}$ cuadrado ajustado es de .074 con el grado de intensidad sobre las variables independientes, al ser superior a .05 es significativo, el error de estándar de estimación es de .338 con un valor significativo pero no es elevado teniendo en cuenta que la muestra no es aleatoria, el valor de Durbin-Watson es de 2.049 detectando de esta forma autocorrelación en la muestra.

Tabla 3. Resumen del modelo

\begin{tabular}{llllll}
\hline Modelo & $\mathbf{R}$ & $\mathbf{R}$ cuadrado & R cuadrado ajustado & Error estándar de la estimación & Durbin-Watson \\
\hline 1 & $.610 a$ & .372 & .074 & .338 & 2.049 \\
\hline
\end{tabular}

a. Predictores: (Constante), L4l, C4c, A4a, E4e, B4b , H4h, G4g, D4c, F4f

b. Variable dependient e: Relación Capit án

En la tabla 4 se trata de ANOVA que trata de un valor de .325 al ser superior a .05 es aceptada la igualdad de medias y no existen diferencias significativas entre los grupos.

Tabla 4. ANOVA

\begin{tabular}{llllll}
\hline Modelo & Suma de cuadrados & GI & Media cuadrática & $\mathbf{F}$ & Sig \\
\hline Regresión & 1.282 & 9 & .142 & 1.249 & .325 \\
\hline Residuo & 2.167 & 19 & .114 & & \\
\hline TOTAL & $\mathbf{3 . 4 4 8}$ & $\mathbf{2 8}$ & & & \\
\hline
\end{tabular}

\subsubsection{Regresión lineal}

En la siguiente tabla se observa las variables analizadas, referidas a los días que la muestra ha contestado que compiten con su equipo, las horas dedicadas fuera de los entrenamientos con sus equipos, las horas dedicadas a otras modalidades de los e-sports y las horas de entrenamiento diarias que le dedican los jugadores a entrenar con su equipo relacionada con la variable dependiente, horas de estudios.

En la tabla 5 se trata de ANOVA que trata de un valor de .696 al ser superior a .05 es aceptada la igualdad de medias y no existen diferencias significativas entre los grupos. 
Tabla 5. ANOVA

\begin{tabular}{|cccccc}
\hline Modelo & Suma de cuadrados & gl & Media cuadrática & F & Sig \\
\hline Regresión & 2.826 & 4 & .707 & .696 & .611 \\
\hline Residuo & 11.174 & 11 & 1.016 & & \\
\hline Total & 14.000 & 15 & & & \\
\hline
\end{tabular}

\section{DISCUSIÓN}

La investigación trataba de conocer en qué medida influyó el profesorado que le impartió clase cuando estudiaba la ESO en estudiantes que practican e-sports. Tras la realización de la regresión y la correlación se puede concluir que, por un lado, existe significación los días que compiten con su equipo, las horas dedicadas fuera de los entrenamientos con sus equipos, las horas dedicadas a otras modalidades de los e-sports y las horas de entrenamiento diarias que le dedican los jugadores a entrenar con su equipo relacionada con la variable dependiente y las horas de estudios influye en su rendimiento académico y en consecuencia en un abandono escolar prematuro. Por otro lado, existe significación también el respecto al profesorado que le dio clase en la ESO, muestra de respeto y trato justo respecto a todo el alumnado, el respeto al profesorado le dio clase durante la ESO, respondía con interés a las intervenciones de los alumnos/as, el respeto al profesorado le dio clase durante la ESO, muestra de respeto y trato justo respecto a todo el alumnado, el respeto al profesorado que le dio clase durante la ESO, organización y preparación de las clases, el respeto al profesorado que le dio clase durante la ESO, las explicaciones relacionadas con ejemplos y la realidad inmediata, el respeto al profesorado que le dio clase durante la ESO, la claridad y precisión de la transmisión de contenidos e ideas en el aula de aprendizaje de los alumnos/as, el respeto al profesorado que le dio clase durante la ESO, el grado de concordancia entre lo explicado y los exámenes realizados) y unido a la relación con los managers y capitanes del equipos de e-sports, relacionado con la socialización.

Las limitaciones sobre el estudio radica en primer lugar la muestra no ha sido aleatoria debido a que he realizado a que como investigador me he integrado como un componente más del equipo, como un jugador más de e-sports, por este motivo las personas analizadas son compañeros míos de equipo bien sea de equipo, de selección andaluza o de selección española de FIFA20 CLUBES PRO, en segundo lugar, otra limitación sobre el estudio es que las personas analizadas tenían libertad de realización sobre el cuestionario online, esto quiere decir que dependiendo de la hora que sea realizado puede influir en la toma de respuestas sobre el cuestionario. 
Por otro lado, como perspectiva de futuro, utilizando muestra de diferentes países y poder hacer una representación cuantitativa sobre los jugadores de e-sports de diferentes países.

\section{CONCLUSIONES}

Tras la realización del estudio se llega a la conclusión que jugar a los e-sports con respecto a las modalidades de CLUBES PRO del videojuego FIFA21 que afecta al rendimiento académico interviene significativamente a un posible abandono escolar, debido al número de horas dedicadas diarias a competir, a las horas empleadas a los entrenamientos oficiales con su club, a las horas dedicas al día a jugar a otras modalidades, los días que compiten afectan todo a las horas dedicadas al estudio.

Por otro lado, se lleva a la conclusión que está altamente relacionado el abandono escolar prematuro con el grado de satisfacción del alumnado hacia el profesorado y en el lado opuesto se encuentra el grado de satisfacción de los jugadores con sus respectivos capitanes, siendo positivamente el grado de satisfacción para continuar en el equipo.

\section{REFERENCIAS BIBLIOGRÁFICAS}

Albert Bandura. (2020). Teoría cognitiva social de la comunicación de masas: Psicología de los medios:

Vol 3, No 3. 2001. https://www.tandfonline.com/doi/abs/10.1207/S1532785XMEP0303 03

Arís Redó, N. (2017). El Síndrome de Burnout en los docentes. Electronic fournal of Research in Education Psychology, 7(18), Article 18. https://doi.org/10.25115/ejrep.v7i18.1324

Ariza-Hernández, M. L. (2017). Influencia de la inteligencia emocional y los afectos en la relación maestro-alumno, en el rendimiento académico de estudiantes de educación superior. Educación y Educadores, 20(2), 193-210. https://doi.org/10.5294/edu.2017.20.2.2

Balladares, P., \& Bersabeh, M. (2018). Relación entre clima social familiar y el rendimiento académico de los estudiantes del cuarto año de educación secundaria de la Institución Educativa 7 de enero Corrales-Tumbes, 2015. Universidad Católica Los Ángeles de Chimbote. http://repositorio. uladech.edu.pe/handle/123456789/2145

Cava, M. J., \& Musitu, G. (2000). Efectos Directos e Indirectos de la Autoestima en el Animo Depresivo. REVISTA MEXICANA DE PSICOLOGIA, 11. 
Chen, Y. (2006). Marketing Innovation. Journal of Economics \& Management Strategy, 15(1), 101-123. https://doi.org/10.1111/j.1530-9134.2006.00093.x

Galanti, A. M. (2020). Cómo generar ambientes positivos y motivantes en el aula. Reflexión Académica en Diseño y Comunicación NoXXIX, Año XVII, Vol. 29, Noviembre 2016, Buenos Aires, Argentina, 96-99.

Humphreys, G. (2019). Sharpening the focus on gaming disorder. Bulletin of the World Health Organization, 97(6), 382-383. https://doi.org/10.2471/BLT.19.020619

INE. (2020). Instituto Nacional de estadística. https://www.ine.es/ss/Satellite?c=INESeccion_C\&p=1254 $\underline{735110672 \& \text { pagename}=\text { ProductosYServicios \%2FPYSLayout } \& \text { cid }=1259925480602 \& \mathrm{~L}=0}$

Jiménez, S. Y., Rubio, E. L., \& Navarro, R. (2016). El apoyo social como factor de protección ante el cyberbullying. La violencia en las relaciones humanas: contextos y entornos protectores del menor, 2016, ISBN 978-84-9044-237-1, págs. 93-114, 93-114. https://dialnet.unirioja.es/servlet/ articulo?codigo $=6078286$

Llamas, C. J. (2015). Perfil profesional de los instructores de clases colectivas en España. AGON: International fournal of Sport Sciences, 5(2), 114-125.

Marcelo, A., \& Luis, J. (2019). Inteligencia Emocional Y Aprendizaje En El Área De Formación Ciudadana Y Cívica En Una Institución Educativa De Santiago, Ica. Repositorio Institucional - UCV. http://repositorio.ucv.edu.pe/handle/UCV/38279

Martinez, J. D. B., Ruiz, L. F. P., \& Hidalgo, E. A. R. (s. f.). Adicción a los Videojuegos. 12.

Rojas-Bermúdez, J. G. (2017). Teoría y técnica sicodramáticas. Punto Rojo Libros.

Salguero, R. T. (2009). Efectos psicosociales de los videojuegos... ISSN, 16.

Sánchez, A., \& Alexander, R. (s. f.). La educación en mediación escolar como escenario de formación ciudadana. 8.

Sancho Álvarez, C., \& Grau Vidal, R. (2013). Diseño y validación de un cuestionario sobre fracaso escolar en alumnado de educación secundaria de un centro de formación de personas adultas. Actualidades Investigativas en Educación, 13(1), 267-291.

Serrano Martínez, L., \& Soler Guillén, Á. (2015). La formación y el empleo de los jóvenes españoles. Fundación BBVA. http://public.ebookcentral.proquest.com/choice/publicfullrecord.aspx?p=4422115

Smetana, J. G., Schlagman, N., \& Adams, P. W. (1993). Preschool Children’s Judgments about Hypothetical and Actual Transgressions. Child Development, 64(1), 202-214. https://doi. org/10.1111/j.1467-8624.1993.tb02904.x 
Teruel Blanco, S. (2017). Estrategia de actividades físicas y recreativas para la transformación conductual en niños de 10 y 11 años que no cumplen los deberes escolares en la comunidad Barrio Obrero. https://repositorio.uho.edu.cu/jspui/handle/uho/2985

Vallejos, M., \& Capa, W. (2010). Video juegos: Adicción y factores predictivos. Avances en Psicología, 18(1), 103-110. https://doi.org/10.33539/avpsicol.2010.n.18.1924

Velasco, C., \& Alejandra, T. (2019). Los videojuegos y su repercusión en el comportamiento de adolescente de 15 años. http://dspace.utb.edu.ec/handle/49000/6521

VFO - NORMATIVA. (2020, marzo 16). http://www.vfospain.com/psn/normativa

VIDEOJUEGOS Y EDUCACIÓN. (2020, marzo 16). https://campus.usal.es/ teoriaeducacion/rev numero $02 / \mathrm{n} 2$ art etxeberria.htm 